\title{
Enhanced piezoelectricity and electromechanical efficiency in semiconducting GaN due to nanoscale porosity
}

Yonatan Calahorra*l, Bogdan Spiridon ${ }^{1}$, Adina Wineman ${ }^{1}$, Tommaso Busolo ${ }^{1}$, Peter Griffin ${ }^{1}$, Piotr K Szewczyk ${ }^{2}$, Tongtong Zhu ${ }^{l}$, Qingshen Jing ${ }^{l}$, Rachel Oliver ${ }^{l}$, Sohini Kar-Narayan ${ }^{* 1}$

${ }^{1}$ Department of Materials Science \& Metallurgy, University of Cambridge, CB3 0FS, Cambridge, UK

${ }^{2}$ International Centre of Electron Microscopy for Materials Science, Faculty of Metals Engineering and Industrial Computer Science, AGH University of Science and Technology, Al. A. Mickiewicza 30, 30059 Kraków, Poland

*Email: yc402@cam.ac.uk, sk568@cam.ac.uk

KEYWORDS: GaN; porous materials; piezoelectric; energy harvesting; atomic force microscopy.

\section{ABSTRACT}

Electrical polarization phenomena in $\mathrm{GaN}$ are important as they have significant impact on the operation of modern day energy efficient lighting and are fundamental to many GaN-based high power and high frequency electronics. Controlling polarization is beneficial for the optimization of these applications. $\mathrm{GaN}$ is also piezoelectric, and therefore mechanical stress and strain are possible handles to control its polarization. Nonetheless, polar semiconductors in general, and $\mathrm{GaN}$ in particular, are weak piezoelectric materials when compared to ceramics, and are therefore not considered for characteristic electromechanical applications such as sensing, actuation and mechanical energy harvesting. Here, we examine the effect of nanoscale porosity on the piezoelectricity of initially conductive GaN. We find that for $40 \%$ porosity, the previously conductive GaN layer becomes depleted, and exhibits enhanced piezoelectricity as measured using piezoresponse force microscopy, as well as by using a mechanical energy harvesting setup. The

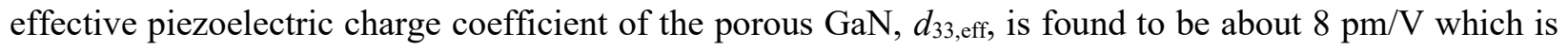


2-3 times larger than bulk GaN. A macroscale device comprising a porous $\mathrm{GaN}$ layer delivered $100 \mathrm{nW} / \mathrm{cm}^{2}$ across a resistive load under a $150 \mathrm{kPa}$ mechanical excitation. We performed finite element simulations to analyze the evolution of the piezoelectric properties with porosity. The simulations suggest that increased mechanical compliance due to porosity gives rise to the observed enhanced piezoelectricity in GaN. Furthermore, the simulations show that for stress-based excitations, the porous GaN electromechanical figure of merit is increased by an order of magnitude and becomes comparable to that of barium titanate piezoceramics. In addition, considering the central role played by $\mathrm{GaN}$ in modern electronics and optoelectronics, our study validates a very promising research direction when considering stress-based electromechanical applications which combine GaN's semiconducting and piezoelectric properties. 


\section{Introduction}

GaN and related III-N technology is becoming increasingly important in electronic and optoelectronic applications, with emerging high-frequency and high-power applications in addition to its established role in energy-efficient lighting. ${ }^{1,2}$ Electrical polarization plays an important role in GaN technology, due to inherent properties of III-N crystal structure, and effects brought about by heterostructure growth. ${ }^{3}$ In particular, III-N materials are piezoelectric, and therefore mechanical strain or stress correspond to development of electrical polarization or field. ${ }^{4,5}$ Interestingly, polarization fields in GaN based light emitting diodes (LEDs) are considered detrimental to LED performance, ${ }^{1,3}$ whereas they are a fundamentally enabling effect in GaN high electron-mobility transistor (HEMT) technology. The latter is implemented in GaN without the need for doping by making use of an interfacial polarization discontinuity. ${ }^{3}$ This indicates the importance and benefits of controlling this phenomenon.

Furthermore, semiconductors which exhibit piezoelectricity make excellent smart/multifunctional materials, as they combine physical characteristics of distinct disciplines, and open up non-trivial routes to control these properties through their coupling. For example, piezoelectric semiconductor nanowires (NWs), and other geometries, have been extensively studied in recent years as active components in applications where mechanical stress or strain controls the electronic/optoelectronic characteristics of semiconductor NW devices (such as their current-voltage characteristics, or light emission intensity). These applications are referred to as piezotronic/photo-piezotronic. ${ }^{6-11}$ III-Ns and non-nitride III-Vs are attracting increased attention in piezotronics ${ }^{5,12}$ due to their mature processing, possible silicon integration and span of desired electronic and optical properties..$^{13-15}$

Porosification has been used as a processing tool to control the optical properties of $\mathrm{GaN}^{16-19}$ as well as its mechanical properties. ${ }^{20}$ Furthermore, several reports demonstrated increased electromechanical coupling following GaN porosification. ${ }^{21-23}$ In this report we examine the effect of nanoscale porosification on the piezoelectric properties of $\mathrm{GaN}$, both at the nanoscale and macroscale, experimentally and through computational modelling. We explore the results specifically in the context of mechanical to electrical energy transfer efficiency and compare the performance of porous $\mathrm{GaN}$ to conventional ferroelectric 
piezoceramics. Our results suggest that porosification is an effective method to increase GaN efficiency as a piezoelectric material and that the performance could be expected to become comparable with ferroelectric piezoceramics, depending on the application. Considering the significant role of GaN in modern electronic technology, this finding has considerable implications for integrated GaN-based electromechanical technologies.

\section{Theoretical background}

\subsection{Piezoelectricity and electromechanical coupling}

Piezoelectricity is the linear coupling of electrical polarization/field $(P / E)$ with mechanical stress/strain $(T / S)$ fields. ${ }^{4}$ Piezoelectricity is manifested in non-centrosymmetric crystals, with 21 out of 32 crystal structures exhibiting piezoelectricity. The linear relations between mechanical stress/strain $(T / S)$ and electrical field/displacement $(E / D)$, are given by

$$
\begin{gathered}
D=\varepsilon_{0} \varepsilon_{r} E \\
T=Y S
\end{gathered}
$$

where $\varepsilon_{0}$ is the vacuum permittivity and $\varepsilon_{r}$ the relative permittivity and $Y$ Young's modulus. The constitutive piezoelectric relations are given by ${ }^{24}$

$$
\begin{gathered}
d_{i j}=\left(\frac{\partial D_{i}}{\partial T_{j}}\right)^{E}=\left(\frac{\partial S_{j}}{\partial E_{i}}\right)^{T} \\
e_{i j}=\left(\frac{\partial D_{i}}{\partial S_{j}}\right)^{E}=-\left(\frac{\partial T_{j}}{\partial E_{i}}\right)^{S} \\
g_{i j}=-\left(\frac{\partial E_{i}}{\partial T_{j}}\right)^{D}=\left(\frac{\partial S_{j}}{\partial D_{i}}\right)^{T} \\
h_{i j}=-\left(\frac{\partial E_{i}}{\partial S_{j}}\right)^{D}=-\left(\frac{\partial T_{j}}{\partial D_{i}}\right)^{S}
\end{gathered}
$$


where the $i, j$ indices identify the piezoelectric coefficient in the $3 \times 6$ matrix representing the piezoelectric tensor (Voigt notation). Subscripts $i\{1-3\}$ and $j\{1-6\}$ represent electrical and mechanical fields correspondingly. The right-hand (left-hand) side equation refers to the converse (direct) piezoelectric effect, and superscripts refer to invariable fields. For example, the direct piezoelectric voltage coefficient, $g_{i j}$, describes the change of the $i$ component of the electric field due to a change in the $j$ component of the mechanical stress field, under constant or zero electrical displacement field - corresponding to open-circuit conditions, i.e., the piezoelectric as a voltage source. Similarly, the converse piezoelectric strain coefficient (also called the piezoelectric charge coefficient), $d_{i j}$, describes the $j$ component of the mechanical strain developed in response to the $i$ component of an applied electric field, under constant or zero mechanical stress.

Atomic force microscopy (AFM) can detect movement on picometer order. The experimental conditions mentioned above correspond to piezoresponse force microscopy (PFM) operation, where the AFM tip is in contact mode, and follows the surface deformation induced by the applied voltage through the tip. Therefore, PFM allows a direct measurement of the out-of-plane $d$ coefficient of the converse piezoelectric effect.

\subsection{Piezoelectricity in GaN}

GaN usually crystallizes in wurtzite structure, ${ }^{3}$ which determines the form of its piezoelectric matrix. The characteristic matrix for wurtzite structures takes the form

$$
d=\left(\begin{array}{cccccc}
0 & 0 & 0 & 0 & -d_{15} & 0 \\
0 & 0 & 0 & -d_{15} & 0 & 0 \\
-d_{31} & -d_{31} & d_{33} & 0 & 0 & 0
\end{array}\right)
$$

with experimental values for $\mathrm{GaN}$ that range $\left|d_{31}\right|=1.5-1.9 \mathrm{pm} / \mathrm{V}, d_{33}=2.5-3.8 \mathrm{pm} / \mathrm{V},\left|d_{15}\right|=$ $1.8-3 \mathrm{pm} / \mathrm{V} \cdot{ }^{25-27}$

As mentioned above, the combination of semiconducting and piezoelectric properties yields interesting piezotronic and photo-piezotronic phenomena. To some extent, this combination is somewhat 
contradictory, as good conductors make poor piezoelectric materials, due to internal screening. Indeed, previous reports studying porous GaN piezoelectricity link it with carrier depletion..$^{21-23}$

\section{Results}

\subsection{Piezoresponse force microscopy}

Porosified and as-grown (non-porous) GaN samples were examined using PFM. The porous layer was about $260 \mathrm{~nm}$ thick. Figure 1a shows a cross section scanning electron microscopy (SEM) image of the porous GaN layer. Figure $1 \mathrm{~b}$ shows a schematic of the layer structure connected for PFM: the only relevant layers are the remnant n-doped GaN (i.e. the part not etched), and the porous layer. The n-doped layer is electrically connected to the AFM stage (where voltage is applied) and the tip (electrical ground) is scanned along the porous layer. The porosity of this sample was estimated as $40 \%$, see Supporting Information Section S1.
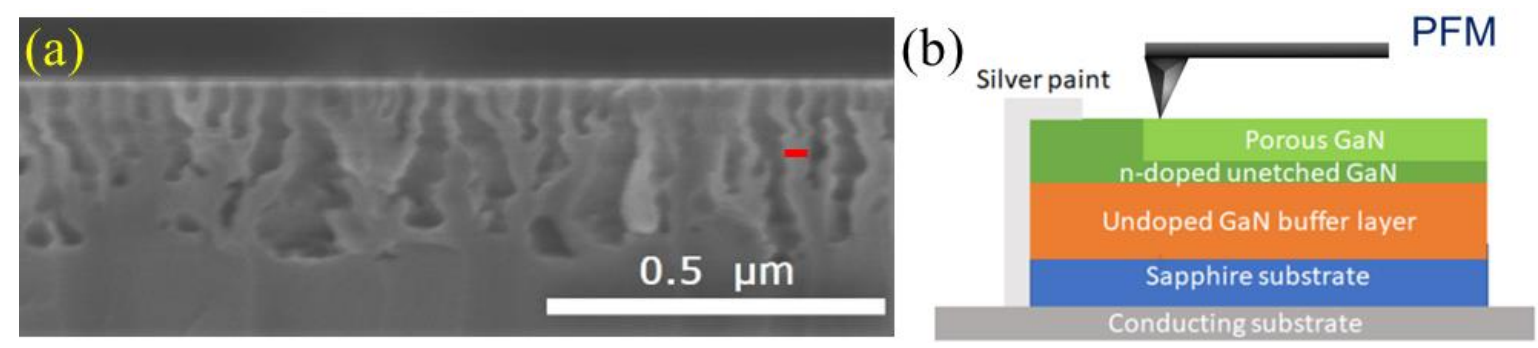

Figure 1 (a) Cross section SEM image of the etched sample. Similar samples were used in the experimental parts of the paper. The red mark indicates $35 \mathrm{~nm}$; (b) Schematic of PFM operation above the layer structure. The "undoped" layer is in practice unintentionally doped (UID) to a level of about $1 \cdot 10^{16} \mathrm{~cm}^{-3}$

PFM was carried out using 2, 4, 6, 8 V AC drive voltages, and the deflections across a $250 \times 500$ $\mathrm{nm}^{2}$ area were recorded (see further details in Methods section). When performing PFM measurements, there are two major parasitic effects to consider: i) background - a parasitic offset signal coming from the instrumentation; ${ }^{28}$ ii) electrostatic contribution to the signal (arising from contact potential differences). ${ }^{29}$ Under the assumption that the background is mostly dependent on the experimental configuration, we measured the sample before etching and subtracted the obtained results from the measurements. The asgrown (un-etched), n-doped, GaN layer is not expected to exhibit piezoelectricity due to screening associated with its high conductivity (carrier density of $5 \cdot 10^{18} \mathrm{~cm}^{-3}$ ), as piezoelectricity sharply decreases 
with conductivity. ${ }^{30,31}$ See more details in Supporting Information S2. We therefore use the following expression for $d_{33}$

$$
d_{33}=d_{\text {measured }}-d_{\text {background }}
$$

In order to minimize the electrostatic contribution, we carried out Kelvin probe force microscopy (KPFM) measurements prior to the PFM measurements, and used the result as a DC bias. ${ }^{29}$ Another consideration is signal calibration: we used a polyvinylidene fluoride-trifluoroethylene (PVDF-TrFE) film made in house, ${ }^{32}$ under the assumption that after poling its piezoresponse is $22 \mathrm{pm} / \mathrm{V} \cdot{ }^{33,34}$ See Supporting Information S2 for details.

Figure 2 PFM deflection vs. AC voltage for the porous GaN sample (blue data points), linearly fitted to yield $8 \mathrm{pm} / \mathrm{V}$ coefficient (slope of the blue dashed line). The range of theoretical bulk values is shown for reference (area). shows calibrated and corrected PFM deflection vs. applied voltage. Each data point represents the spatial mean and standard deviation of a single PFM scan, obtained

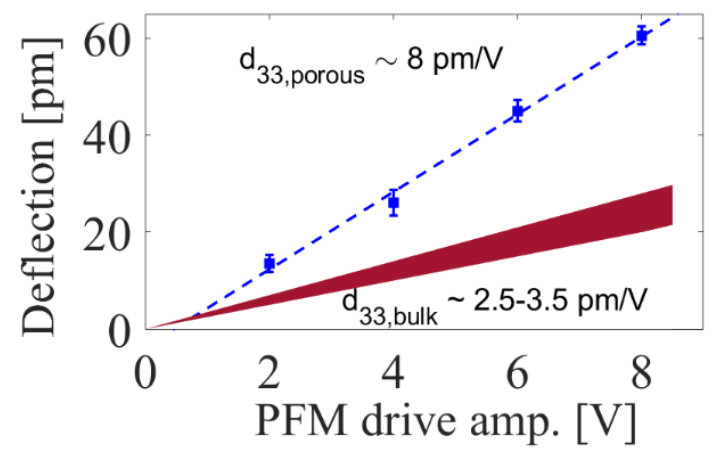

Figure 2 PFM deflection vs. AC voltage for the porous GaN sample (blue data points), linearly fitted to yield 8 pm/V coefficient (slope of the blue dashed line). The range of theoretical bulk values is shown for reference (area).

in a resolution of $256 \times 512$ points (scans are shown in Supporting Information Figure S6). The piezoelectric coefficient was obtained by the slope of linear fitting of the data points. This yielded a porous GaN coefficient of $d_{33}=8 \pm 1.6 \mathrm{pm} / \mathrm{V}$. The relatively large error margin was due to taking into account a $20 \%$ uncertainty in the $d_{33}$ coefficient value of PVDF-TrFE used here for PFM calibration. ${ }^{32}$ In any case, the standard deviations of the PFM measurements are smaller than $20 \%$. The corresponding curve for bulk 
GaN (filled area calculated based on known coefficient range) is also shown for reference. Our results suggest that for $\sim 40 \%$ porosity, the effective $d_{33}$ is about three times that of bulk GaN.

\subsection{Mechanical Energy Harvesting}

We further examined the macroscale electromechanical performance of the etched GaN layer. Current-voltage $(I-V)$ measurements of the sample did not show any measurable current (data not shown), and therefore piezotronic activity ${ }^{10}$ was not relevant for this sample. Considering that the starting material was a $5 \cdot 10^{18} \mathrm{~cm}^{-3} \mathrm{Si}$ doped GaN layer, this result indicates the layer became depleted.

Figure 3 shows the short-circuit current measured while the layer was periodically tapped by a blunt indenter applying $1.5 \mathrm{~N}$ at $2 \mathrm{~Hz}$. The indenter was roughly the size of the contact $\left(2 \mathrm{x} 5 \mathrm{~mm}^{2}\right)$, leading to an assessment of the applied stress of $150 \mathrm{kPa}$. Noticeably, the approach and retract (compress and release) peaks are not symmetrical. The experiment was repeated with a set of resistive loads spanning $1 \mathrm{k} \Omega-100$ $\mathrm{M} \Omega$. A peak-finding algorithm was used to locate the highest approach and retract peaks (as seen in Figure 3b), and the RMS value was calculated accordingly. Although the mechanical excitation was applied at 2
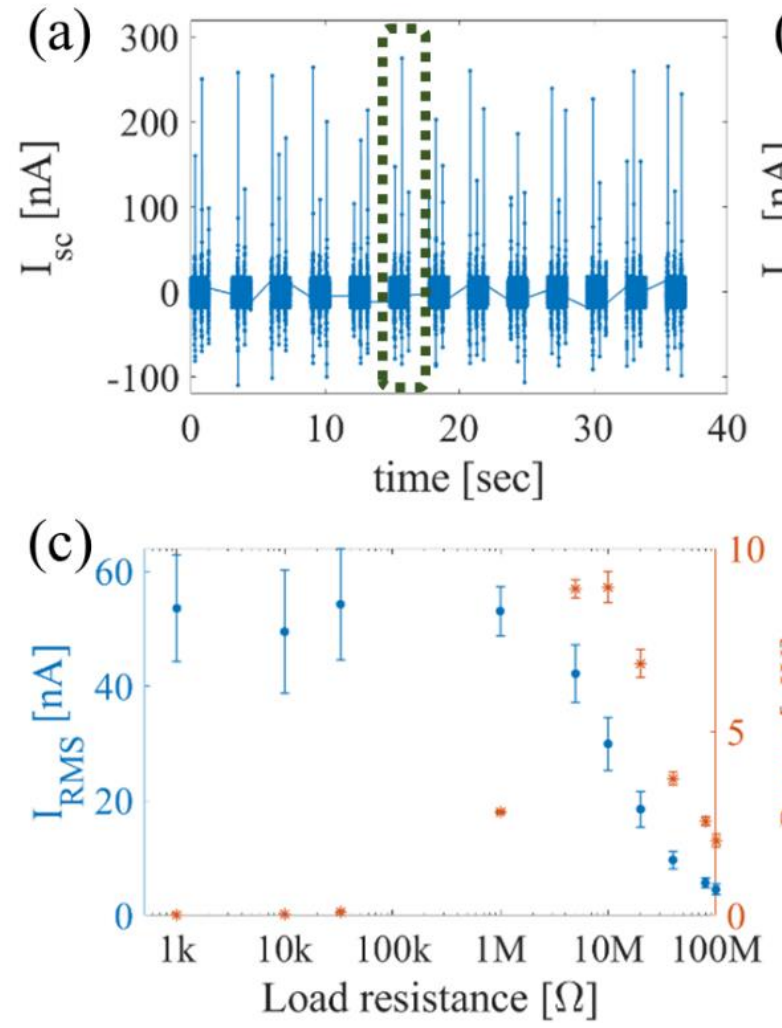

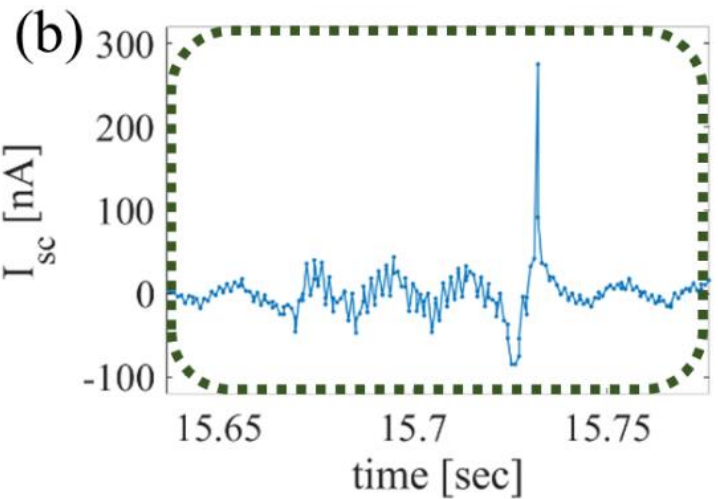

(d)

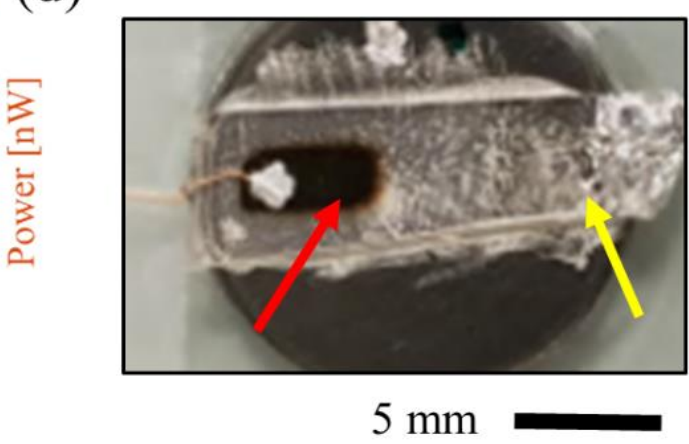


$\mathrm{Hz}$, only the actual duration of contact is relevant for power transfer, and so the signal was integrated around the two prominent peaks to find RMS current.

Figure 3. Generated current measured during repetitive loading of the porous GaN layer, by applying $1.5 \mathrm{~N} / 150 \mathrm{kPa}$ : (a) measured short-circuit current. The interrupted intervals are due to data recording and storage, the excitation was continuous; (b) zoom into one of the peak regions showing compress and release peaks. The prominent peaks were identified and used to calculate RMS current as well as the power; (c) RMS current (blue dots, left axis) as a function of load resistance and the corresponding power (orange asterisk, right axis); (d) top view of the device, where the left hand side arrow (red) points to the sputtered top contact and force application location, and the right hand side arrow (yellow) points to the bottom contact - effectively touching the un-etched part of the sample.

The maximum power transfer from the $150 \mathrm{kPa}$ stress is about $9 \mathrm{nW}$, obtained for loads of 5 and $10 \mathrm{M} \Omega$ (indicating the optimized value would be slightly higher). Considering the contact area, this corresponds to an area density of $100 \mathrm{nW} / \mathrm{cm}^{2}$ and a volume density of about $4 \mathrm{~mW} / \mathrm{cm}^{3}$, accounting for about $260 \mathrm{~nm}$ of porous layer thickness. These results (for area density) are in good agreement with previous porous GaN energy harvesting experiments reported in recent years. ${ }^{22,23}$

\subsection{Finite element simulation - COMSOL}

In order to obtain a deeper understanding of the electromechanical effects at play in porous GaN, we carried out COMSOL simulations on GaN structures with varying porosity. As shown in Figure 4, we simulated a hexagonal unit cell (100 nm long diagonal dimension), with periodic boundary conditions effectively a thin film. The pore was cylindrical with a varying diameter, enabling the presentation of results as a function of porosity, which is calculated as the volumetric ratio of air to GaN. This design is inspired by the observed morphology (Figure 2). The total thickness was $500 \mathrm{~nm}$ and the pore depth was $400 \mathrm{~nm}$. The structure was capped by a $10 \mathrm{~nm} \mathrm{GaN}$ layer to simplify the application of forces to the structure (constant stress). The pore diameter was varied between 1 and $81 \mathrm{~nm}$, corresponding to porosity of up to

(a)

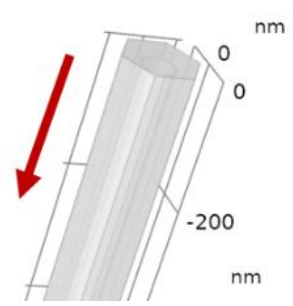

(b)

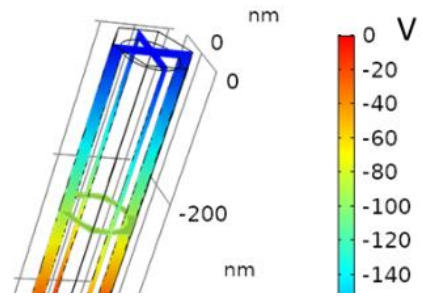

Figure 4 COMSOL simulations: (a) the simulated geometry, a periodic GaN structure, with an embedded pore of varying diameter. The arrow shows the direction of $5 \mathrm{GPa}$ stress applied to the structure; (b) the piezoelectric potential developed following the applied pressure for the largest porosity value of $\sim 42 \%$. 
$42 \%$. The default GaN parameters were used with the addition of the piezoelectric coefficients $d_{31}=1.5$, $d_{33}=3.8, d_{15}=1.8 \mathrm{pm} / \mathrm{V}$ (with the appropriate sign applied). A relatively high $d_{33}$ value was chosen to increase the simulated effect. Figure $4 \mathrm{~b}$ shows the potential distribution for the largest pore size.

Figure 5 shows the 'raw' output of the simulation: the top surface displacement and potential difference across the simulated element as a function of porosity - for a given load of $5 \mathrm{GPa}$. The results are straightforward - deflection and voltage both increase considerably with porosity (two, and three-fold at $42 \%$, correspondingly). This would be expected, as a porous material is more compliant ${ }^{35,36}$, and the remaining material produces the open-circuit voltage. This raises several interesting questions, for example what are the effective piezoelectric constants? and what is the electromechanical coupling efficiency as a function of porosity?

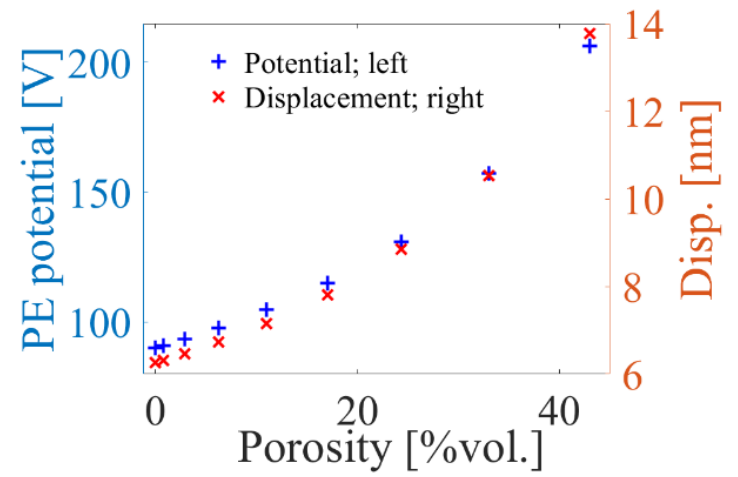

Figure 6 Simulated displacement (right hand side) and piezoelectric potential (left hand side) in the studied structure as a function of volume porosity for $5 \mathrm{GPa}$ stress.

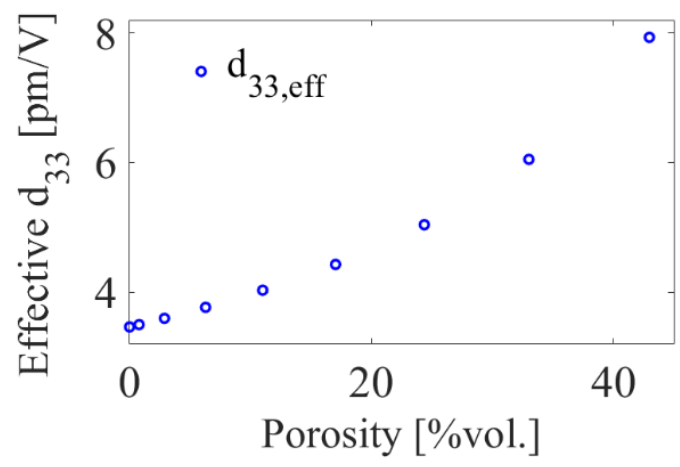

Figure 5. Simulated effective $d_{33}$ as a function of structure porosity.

To examine these questions, we calculated several quantities based on the simulation results. First, we consider the effective out-of-plane piezoelectric coefficient. The electrical boundary conditions for the model were open-circuit, therefore calculating $d$ coefficients is not straightforward and requires calculating 
the piezoelectric voltage coefficient, $g$, first (see Equation 5), since the $d$ coefficient is defined under constant electric field. We have recently demonstrated this procedure experimentally. ${ }^{37}$ The $g$ coefficient is the ratio between the developed electric field and the applied stress. The relation between the two coefficients is given by $d=\varepsilon g$, and this allows us to calculate $d$, as shown in Figure 6 . The piezoelectric coefficient follows the trend seen in the displacement and piezoelectric voltage - an increase with porosity. We point out towards two interesting observations - the highest value found is $7.93 \mathrm{pm} / \mathrm{V}$ for $42 \%$ porosity, in very good agreement with the experimental value found using PFM for about $40 \%$ porosity. Also, the

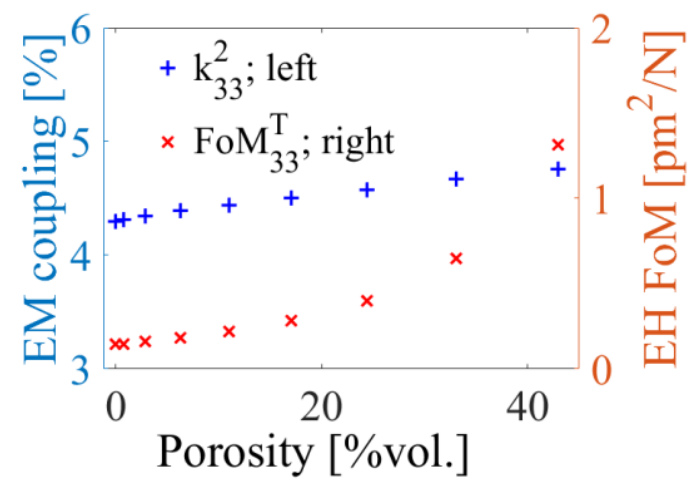

Figure 7 Simulation extracted $\mathrm{k}^{\wedge} 2$ (blue + , left) and FoM (red x, right) as a function of porosity. Right hand side axis units are $10^{-12} \mathrm{~m}^{2} / \mathrm{N}$.

zero porosity value, $\sim 3.4 \mathrm{pm} / \mathrm{V}$, is actually lower than the input $d_{33}(3.8 \mathrm{pm} / \mathrm{V})$. As discussed below in detail, this is a demonstration of the bulk confinement effect, where the lack of it contributes to the increase in piezoelectric coefficients of nanomaterials. ${ }^{38}$

Next, we examine the energy conversion efficiency. We consider two figures of merit for the electromechanical (EM) coupling: one is the electromechanical coupling efficiency coefficient, $k^{2}$, defined as the ratio between stored electrical energy and input mechanical energy; the other is the stress-based energy harvesting figure of merit (EH FoM) which is given by the product $d \cdot g{ }^{39,40}$. These quantities take the form

$$
k_{i j}^{2}=\frac{d_{i j}^{2}}{\varepsilon_{i i}^{T} s_{j j}^{E}}
$$




$$
F_{o M} M_{i j}^{T}=d_{i j} \cdot g_{i j}=\frac{d_{i j}^{2}}{\varepsilon_{i i}^{T}}
$$

Noticeably, the difference between the two figures of merit is the lack of the compliance term $\left(s_{j j}^{E}\right)$ in the denominator in $F o M_{i j}^{T}$, which means that softer materials do not suffer an efficiency penalty, making compliant materials such as PVDF good sensors/energy harvesters ${ }^{39,41,42}$ - when the excitation is based on stress and not strain.

Figure 7 Simulation extracted $\mathrm{k}^{\wedge} 2$ (blue + , left) and FoM (red $\mathrm{x}$, right) as a function of porosity. shows the two figures of merit calculated based on the simulation, as a function of porosity. For the efficiency calculation, we considered the input mechanical energy as the product of the applied force and the displacement, and the stored electrostatic energy was calculated directly by COMSOL; for FoM $M_{i j}^{T}$ calculation, we approximated the effective permittivity as a linear function of porosity volume fraction $(\eta)$, $\varepsilon_{e f f}=\eta \varepsilon_{G a N} \varepsilon_{0}$, and used Equation 10

The results indicate a significant distinction between the two figures of merit: while the electromechanical coupling efficiency remains nearly unchanged with porosity (slight increase), $F o M_{i j}^{T}$ increases nearly 10 fold. This suggests that porosification is a promising strategy for increasing efficiency in stress based piezoelectric applications.

\section{Discussion}

\subsection{Transition of GaN from conductive to an active piezoelectric}

The porosification process described here is based on conductivity, and its efficiency increases with doping/conductivity. We consider two routes for the transition from a conducting starting material to an un-screened piezoelectric material: i) the remaining material has increased effective resistance due to geometry (dimensions or connectivity) ii) the remaining nanostructure becomes depleted, due to increased surface area (and hence surface depletion) and possibly due to size effects (quantum confinement). We have observed that similar etching profiles obtained from samples of initially higher doping levels then reported 
here, result in structures that are still conducting. This is an indication that the geometry and connectivity aspects alone, do not explain the significant decrease in conductivity and onset of piezoelectricity. We therefore focus on the second possibility, size induced depletion.

The onset of carrier depletion in $\mathrm{GaN}$ due to porosification has been considered to be an important mechanism in previous work regarding porous GaN piezoelectricity. ${ }^{23}$ The relevant feature size in our material, as seen in Figure 1a, is about $35 \mathrm{~nm}$, although some features are thinner than this. Previous studies have shown that this size falls in the range of critical thickness (in form of GaN nanowire diameter) resulting in a significant decrease in conductivity due to surface depletion. ${ }^{43,44}$ This is a general outcome of size and geometry in semiconductors and not unique to $\mathrm{GaN} .{ }^{45}$ Therefore, we agree with previous interpretations that size related carrier depletion is responsible for the onset of piezoelectricity in the initially doped GaN. In the following section, we discuss the emergence of piezoelectricity in porous GaN which is considerably larger than bulk GaN.

\subsection{Increased piezoresponse of porous GaN}

Lateral mechanical clamping reduces the ability of a piezoelectric thin film to react to an excitation. It has been demonstrated that nanostructures experience reduced clamping, and an increased piezoelectric response. ${ }^{38}$ Lateral clamping is expressed by ${ }^{46}$

$$
d_{33}^{E f f}=d_{33}^{M a t r i x}-\frac{2 s_{13}}{s_{11}+s_{12}} d_{31}^{\text {Matrix }}
$$

where $s_{i j}$ are compliance matrix elements, and superscripts indicate matrix elements and effective/measured values. The matrix elements we used in our COMSOL simulation are $d_{33}^{\text {Matrix }}=3.8$, $d_{31}^{\text {Matrix }}=-1.5 \mathrm{pm} / \mathrm{V}$ and $s_{11}=3.03, s_{12}=-0.659, s_{13}=-0.322 \mathrm{GPa}^{-1}$. Using Eq. (9), this yields

$d_{33}^{E f f}=3.39 \mathrm{pm} / \mathrm{V}$, which is in very good agreement to the simulation result (Figure 6 at zero porosity), indicating that the simulation captures this effect successfully. Logic dictates that substrate clamping removal will allow the measured/simulated coefficient to be only as high as the matrix coefficient $(3.8$ 
$\mathrm{pm} / \mathrm{V}$ in our case). Therefore, it does not explain the full extent of the results as they are manifested in PFM and simulation. The conclusion therefore is that the overall structure of the porous material, and the increased compliance in particular, are responsible for the results.

\subsection{Comparison to porous ceramics}

As mentioned above, porosity as a tool to enhance piezoelectricity has been studied mostly in the context of ceramics, ${ }^{40,47-49}$, polymers, ${ }^{50}$ and even as a route to induce piezoelectricity in originally centrosymmetric $2 \mathrm{D}$ materials $\mathrm{s}^{51,52}$ - in addition to GaN. ${ }^{22,23}$ When considering ceramics, and ferroelectrics in general, a poling process is necessary for dipole alignment and onset of measurable piezoelectricity. This process is dependent upon the ability to efficiently apply an electric field across the ferroelectric material. The ability to efficiently pole a porous structure is found to depend both on the total porosity, and the pore shape, where aligned/directional pores offer better efficiency in poling. ${ }^{47,}{ }^{49}$ This then affects the piezoelectric properties of these structures, such that the increased compliance (and subsequent increased piezoelectricity) competes with the reduced poling efficiency, brought by inefficient electric field distribution..$^{40,48}$

Conversely, GaN is not a ferroelectric, and once grown, it does not require poling. Therefore, the only effect at play is the increase in mechanical compliance. As seen above, the electromechanical coupling efficiency is mostly unaffected by the porosity and remains nearly constant, but the stress-based figure of merit is not diminished with the reduced compliance. It is interesting to compare the simulation results presented here to the poling-piezoelectric simulations presented by Roscow and co-workers. ${ }^{40}$ For porous barium titanate simulated from zero to $40 \%$ porosity (accounting for poling and electromechanical properties), $d_{33}$ was reduced by about a half, from $\sim 140$ to $\sim 80 \mathrm{pm} / \mathrm{V}$, the electromechanical coupling efficiency $\left(\mathrm{k}^{2}\right)$ decreased from 0.45 to 0.25 , and $F o M_{i j}^{T}$ was found to increase from $\sim 1.5$ to $\sim 1.910^{-12} \mathrm{~m}^{2} / \mathrm{N}$. These results are a manifestation of the competing mechanisms discussed above.

In comparison, for the same range of porosity in $\mathrm{GaN}, d_{33}$ increased from 3.4 to $8 \mathrm{pm} / \mathrm{V}$ (remarkably close to the experimental value), $\mathrm{k}^{2}$ was slightly increased, and $F o M_{i j}^{T}$ increased 10 fold from 
$\sim 0.12$ to $\sim 1.210^{-12} \mathrm{~m}^{2} / \mathrm{N}$ - close to the barium titanate. This is a striking result, indicating that for stressbased applications porous GaN can perform almost as well as barium titanate - a material with a piezoelectric charge coefficient (d) about 50 times higher, when comparing bulk forms.

\section{Conclusions}

We studied the effect of porosity on the piezoelectric properties of a layer of conductive GaN. $40 \%$ volume porosification was found to deplete the structure, resulting in the onset of piezoelectricity. This was characterized at the nanoscale by PFM, where a piezoelectric coefficient 2-3 times larger than bulk GaN was found (8 compared to $2.5-3.5 \mathrm{pm} / \mathrm{V}$ ). Macroscale energy harvesting experiments demonstrated that the porous $\mathrm{GaN}$ layer operates as a piezoelectric transducer, with maximal power densities of $100 \mathrm{nW} / \mathrm{cm}^{2}$ transferred to a resistive load of $\sim 10 \mathrm{M} \Omega$, under $150 \mathrm{kPa}$ load.

We have performed detailed finite element simulations to examine the effect of porosity on the piezoelectric coefficient and efficiencies. The simulations suggest that the electromechanical coupling coefficient $\left(k^{2}\right)$ remains nearly constant with porosity - indicating it is determined by intrinsic material properties. Conversely, the stress-based electromechanical figure of merit, which is independent of effective compliance, is increased 10 fold for $40 \%$ porosity, bringing the porous $\mathrm{GaN}$ to a level comparable to piezoelectric ceramic such as barium titanate.

Our combined modelling/experimental study is promising when considering porous $\mathrm{GaN}$ as an efficient piezoelectric material, and it opens up interesting avenues for integration of electromechanical applications within dominant microelectronic processes and technologies.

\section{Materials and Methods}

\subsection{GaN growth and porosification}

n-doped GaN was grown on sapphire by metal-organic vapor phase epitaxy (MOVPE) and porosified in a fashion similar to previous studies ${ }^{18,19}$. Briefly, a layer of n-doped GaN $\left(\sim 5 \cdot 10^{18} \mathrm{~cm}^{-3} ; 1.5\right.$ $\mu \mathrm{m})$ was grown on top of a non-intentionally doped low-dislocation density GaN pseudo-substrate ${ }^{53}$. The sample was immersed in oxalic acid $(0.25 \mathrm{M})$ with the nitride surface of the doped layer exposed and a DC potential of 8-14 $\mathrm{V}$ applied between the sample (thus determining the level of porosification) and an inert 
platinum counter electrode. The sample used in AFM and energy harvesting measurements were etched using $14 \mathrm{~V}$ bias, for a duration such that the $1.5 \mu \mathrm{m}$ doped layer was etched to about a fifth of its thickness (see Supporting Information S1 and Figure S1). The porosification process does not affect the composition or crystallinity of the remaining material. ${ }^{18,19}$

\subsection{Atomic force microscopy (AFM)}

AFM measurements were carried out using a Bruker Dimension Icon, and the results were analyzed using Nanoscope Analysis 1.9 (Bruker). For PFM and KPFM MESP-RC-V2 tips were used while for quantitative nanoscale mechanical mapping (QNM) the peak-force mode standard ScanAsyst-Air tip was used. PFM was performed by applying different AC voltage amplitudes at a frequency of $125 \mathrm{kHz}$ which is away from any contact resonance. This ScanAsyst-Air tip is not suitable for quantitative mechanical analysis of materials as hard as GaN, leading to the QNM results treated as qualitative (see Supporting Information S2).

\subsection{Device fabrication and energy harvesting}

The device was fabricated as follows. The sample was mounted on a round (15 $\mathrm{mm}$ in diameter) metallic stub (used initially for AFM - Figure 1 (a) Cross section SEM image of the etched sample. Similar samples were used in the experimental parts of the paper. The red mark indicates $35 \mathrm{~nm}$; (b) Schematic of PFM operation above the layer structure.), which was electrically connected to the conductive GaN layer. This served as a bottom electrode. The top electrode was sputtered with gold ( $\sim 80$ $\mathrm{nm}$ ), made on the etched part of the sample using a shadow mask with a size of about $0.25 \times 0.5 \mathrm{~cm}^{2}$ (Figure 3).

Short-circuit and loaded mechanical energy harvesting experiments were carried out using a linear motor setup, set to tap the device (max. $1.5 \mathrm{~N}$ at $2 \mathrm{~Hz}$ ). The generated current was measured using a picoammeter (Keithley 6487). The device was mounted on a force sensor (AEP Transducers TCA type, sshaped), and the force was recorded separately from the electrical output.

\subsection{COMSOL Multiphysics}


COMSOL Multiphysics v. 5.2 was used to simulate the piezoelectricity of the porous GaN relying on the Piezoelectricity module. GaN was used with an addition to a user-defined $d$ matrix, as discussed above, since GaN is not in the default library of piezoelectric materials. The simulation was run calculating stress-charge quantities, and the electrical connectivity was open-circuit, hence $g$ quantities were directly extracted from simulation results, yielding $d$ as described above.

\section{Acknowledgments}

Y.C. and S.K-N. are grateful for support from ERC Starting Grant (Grant No. ERC-2014-STG639526, NANOGEN), as well as Henry Royce Institute - Cambridge Equipment grant EP/P024947/1 and the Centre of Advanced Materials for Integrated Energy Systems "CAM-IES" grant EP/P007767/1. T.B. acknowledges funding from the EPSRC Cambridge NanoDTC, EP/G037221/1. Q.J. acknowledges support from the Centre of Advanced Materials for Integrated Energy Systems "CAM-IES" grant EP/P007767/1 R.A.O. and Y.C. acknowledge funding from the EPSRC under EP/M010589/1 and the Cambridge University Impact Acceleration Account. P.H.G and B.F.S. have been supported by EPSRC Centres for Doctoral Training under EP/L015455/1 and EP/L015889/1 respectively. B.F.S. gratefully acknowledges support from the Cambridge Commonwealth European and International Trust. P.K.S. acknowledges the funding from COST Action CA17107 (CONTEXT) funded by the European Commission and POWER 3.5 (POWR.03.05.00-00-Z307/17-00) granted by the European Union.

Data availability: Supporting data for this paper is available at the DSpace@Cambridge data repository (https://doi.org/xx.xxxxx/CAM.xxxxx).

Supporting Information. Technical details about GaN porosification process and resulting porosity, as well as about PFM calibration and measurements are available in Supporting Information.

\section{References}


(1) Humphreys, C. J. Solid-state lighting. Mrs Bulletin 2008, 33 (4), 459-470, DOI: $10.1557 / \mathrm{mrs} 2008.91$.

(2) Mishra, U. K.; Shen, L.; Kazior, T. E.; Wu, Y. F. GaN-Based RF power devices and amplifiers. Proceedings of the Ieee 2008, 96 (2), 287-305, DOI: 10.1109/jproc.2007.911060.

(3) Wood, C.; Jena, D.; SpringerLink (Online service), Polarization Effects in Semiconductors

From Ab Initio Theory to Device Applications. Springer Science+Business Media, LLC,: Boston, MA, 2008. http://dx.doi.org/10.1007/978-0-387-68319-5.

(4) Curie, J.; Curie, P. Développement par compression de l'électricité polaire dans les cristaux hémièdres à faces inclinées. Bulletin de minéralogie 1880, 3, 4.

(5) Calahorra, Y.; Ou, C. L.; Boughey, C.; Kar-Narayan, S. Piezoelectric Semiconducting Nanowires. Nanowires for Energy Applications 2018, 98, 445-478, DOI: 10.1016/bs.semsem.2018.02.002.

(6) Wang, X. D.; Zhou, J.; Song, J. H.; Liu, J.; Xu, N. S.; Wang, Z. L. Piezoelectric field effect transistor and nanoforce sensor based on a single $\mathrm{ZnO}$ nanowire. Nano Letters 2006, 6 (12), 27682772, DOI: $10.1021 / \mathrm{nl} 061802 \mathrm{~g}$.

(7) Wang, Z. L. Piezopotential gated nanowire devices: Piezotronics and piezo-phototronics. Nano Today 2010, 5 (6), 540-552, DOI: 10.1016/j.nantod.2010.10.008.

(8) Wang, L. F.; Liu, S. H.; Feng, X. L.; Xu, Q.; Bai, S.; Zhu, L. P.; Chen, L. B.; Qin, Y.; Wang, Z. L. Ultrasensitive Vertical Piezotronic Transistor Based on ZnO Twin Nanoplatelet. Acs Nano 2017, 11 (5), 4859-4865, DOI: 10.1021/acsnano.7b01374.

(9) Keil, P.; Fromling, T.; Klein, A.; Rodel, J.; Novak, N. Piezotronic effect at Schottky barrier of a metal-ZnO single crystal interface. Journal of Applied Physics 2017, 121 (15), DOI: $10.1063 / 1.4981243$.

(10) Calahorra, Y.; Husmann, A.; Bourdelain, A.; Kim, W.; Vukajlovic-Plestina, J.; Boughey, C.; Jing, Q.; i Morral, A. F.; Kar-Narayan, S. Highly sensitive piezotronic pressure sensors based on undoped GaAs nanowire ensembles. Journal of Physics D: Applied Physics 2019.

(11) Keil, P.; Trapp, M.; Novak, N.; Fromling, T.; Kleebe, H. J.; Rodel, J. Piezotronic Tuning of Potential Barriers in $\mathrm{ZnO}$ Bicrystals. Advanced Materials 2018, 30 (10), DOI: 10.1002/adma.201705573.

(12) Calahorra, Y.; Kar-Narayan, S. Piezoelectricity in non-nitride III-V nanowires: Challenges and opportunities. Journal of Materials Research 2018, 33 (6), 611-624, DOI: 10.1557/jmr.2018.29.

(13) Vukajlovic-Plestina, J.; Kim, W.; Dubrovski, V. G.; Tutuncuoglu, G.; Lagier, M.; Potts, H.; Friedl, M.; Morral, A. F. I. Engineering the Size Distributions of Ordered GaAs Nanowires on Silicon. Nano Letters 2017, 17 (7), 4101-4108, DOI: 10.1021/acs.nanolett.7b00842.

(14) Friedl, M.; Cerveny, K.; Weigele, P.; Tutuncuoglu, G.; Marti-Sanchez, S.; Huang, C. Y.; Patlatiuk, T.; Potts, H.; Sun, Z. Y.; Hill, M. O.; Guniat, L.; Kim, W.; Zamani, M.; Dubrovskii, V. G.; Arbiol, J.; Lauhon, L. J.; Zumbuhl, D. M.; Morral, A. F. I. Template-Assisted Scalable Nanowire Networks. Nano Letters 2018, 18 (4), 2666-2671, DOI: 10.1021/acs.nanolett.8b00554. (15) Cutaia, D.; Moselund, K. E.; Schmid, H.; Borg, M.; Olziersky, A.; Riel, H.; Ieee. Complementary III-V Heterojunction Lateral NW Tunnel FET Technology on Si. 2016 Ieee Symposium on Vlsi Technology 2016.

(16) Yerino, C. D.; Zhang, Y.; Leung, B.; Lee, M. L.; Hsu, T. C.; Wang, C. K.; Peng, W. C.; Han, J. Shape transformation of nanoporous $\mathrm{GaN}$ by annealing: From buried cavities to nanomembranes. Applied Physics Letters 2011, 98 (25), DOI: 10.1063/1.3601861. 
(17) Chen, D. T.; Xiao, H. D.; Han, J. Nanopores in GaN by electrochemical anodization in hydrofluoric acid: Formation and mechanism. Journal of Applied Physics 2012, 112 (6), DOI: $10.1063 / 1.4752259$.

(18) Zhu, T. T.; Liu, Y. J.; Ding, T.; Fu, W. Y.; Jarman, J.; Ren, C. X.; Kumar, R. V.; Oliver, R. A. 0-Wafer-scale Fabrication of Non-Polar Mesoporous GaN Distributed Bragg Reflectors via Electrochemical Porosification. Scientific Reports 2017, 7, DOI: 10.1038/srep45344.

(19) Griffin, P.; Zhu, T. T.; Oliver, R. Porous AlGaN-Based Ultraviolet Distributed Bragg Reflectors. Materials 2018, 11 (9), DOI: 10.3390/ma11091487.

(20) Huang, S. J.; Zhang, Y.; Leung, B.; Yuan, G.; Wang, G.; Jiang, H.; Fan, Y. M.; Sun, Q.; Wang, J. F.; Xu, K.; Han, J. Mechanical Properties of Nanoporous GaN and Its Application for Separation and Transfer of GaN Thin Films. Acs Applied Materials \& Interfaces 2013, 5 (21), 11074-11079, DOI: 10.1021/am4032345.

(21) Kang, J. H.; Ebaid, M.; Jeong, D. K.; Lee, J. K.; Ryu, S. W. Efficient energy harvesting of a GaN p-n junction piezoelectric generator through suppressed internal field screening. Journal of Materials Chemistry C 2016, 4 (15), 3337-3341, DOI: 10.1039/c6tc00040a.

(22) Waseem, A.; Jeong, D. K.; Johar, M. A.; Kang, J. H.; Ha, J. S.; Lee, J. K.; Ryu, S. W. Enhanced piezoelectric output of $\mathrm{NiO}$ /nanoporous $\mathrm{GaN}$ by suppression of internal carrier screening. Semiconductor Science and Technology 2018, 33 (6), DOI: 10.1088/1361-6641/aabf5f. (23) Kang, J. H.; Jeong, D. K.; Ha, J. S.; Lee, J. K.; Ryu, S. W. Enhanced performance of a GaN piezoelectric nanogenerator with an embedded nanoporous layer via the suppressed carrier screening effect. Semiconductor Science and Technology 2017, 32 (2), DOI: 10.1088/13616641/32/2/025001.

(24) Damjanovic, D. Ferroelectric, dielectric and piezoelectric properties of ferroelectric thin films and ceramics. Reports on Progress in Physics 1998, 61 (9), 1267-1324, DOI: 10.1088/00344885/61/9/002.

(25) Minary-Jolandan, M.; Bernal, R. A.; Kujanishvili, I.; Parpoil, V.; Espinosa, H. D. Individual GaN Nanowires Exhibit Strong Piezoelectricity in 3D. Nano Letters 2012, 12 (2), 970-976, DOI: $10.1021 / \mathrm{nl} 204043 \mathrm{y}$.

(26) Hanada, T. Basic Properties of $\mathrm{ZnO}, \mathrm{GaN}$, and Related Materials. Oxide and Nitride Semiconductors: Processing, Properties, and Applications 2009, 12, 1-19.

(27) Guy, I. L.; Muensit, S.; Goldys, E. M. Extensional piezoelectric coefficients of gallium nitride and aluminum nitride. Applied Physics Letters 1999, 75 (26), 4133-4135, DOI: 10.1063/1.125560. (28) Jungk, T.; Hoffmann, A.; Soergel, E. Consequences of the background in piezoresponse force microscopy on the imaging of ferroelectric domain structures. Journal of Microscopy-Oxford 2007, 227 (1), 72-78, DOI: 10.1111/j.1365-2818.2007.01783.x.

(29) Kim, S.; Seol, D.; Lu, X. L.; Alexe, M.; Kim, Y. Electrostatic-free piezoresponse force microscopy. Scientific Reports 2017, 7, DOI: 10.1038/srep41657.

(30) Scrymgeour, D. A.; Hsu, J. W. P. Correlated piezoelectric and electrical properties in individual ZnO nanorods. Nano Letters 2008, 8 (8), 2204-2209, DOI: 10.1021/n1080704n.

(31) Ogawa, T.; Oikawa, H.; Kojima, A. Decrement of Piezoelectric Constants Caused by Screening Effect of Conduction Electrons on the Effective Charge of CdS Crystals. Japanese Journal of Applied Physics 1971, 10 (5), 593-599, DOI: 10.1143/jjap.10.593.

(32) Whiter, R. A.; Calahorra, Y.; Ou, C. L.; Kar-Narayan, S. Observation of ConfinementInduced Self-Poling Effects in Ferroelectric Polymer Nanowires Grown by Template Wetting. Macromolecular Materials and Engineering 2016, 301 (9), 1016-1025, DOI: 10.1002/mame.201600135. 
(33) Choi, Y.-Y.; Hong, J.; Hong, S.; Song, H.; Cheong, D.-S.; No, K. Nanoscale piezoresponse of $70 \mathrm{~nm}$ poly(vinylidene fluoride-trifluoro-ethylene) films annealed at different temperatures. physica status solidi (RRL) - Rapid Research Letters 2010, 4 (3-4), 94-96, DOI: 10.1002/pssr.201004009.

(34) Calahorra, Y.; Smith, M.; Datta, A.; Benisty, H.; Kar-Narayan, S. Mapping piezoelectric response in nanomaterials using a dedicated non destructive scanning probe technique. Nanoscale 2017, 9, 8, DOI: 10.1039/C7NR06714C.

(35) Fleck, N. A.; Otoyo, H.; Needleman, A. INDENTATION OF POROUS SOLIDS. International Journal of Solids and Structures 1992, 29 (13), 1613-1636, DOI: 10.1016/00207683(92)90012-i.

(36) Chen, X.; Xiang, Y.; Vlassak, J. J. Novel technique for measuring the mechanical properties of porous materials by nanoindentation. Journal of Materials Research 2006, 21 (3), 715-724, DOI: $10.1557 / \mathrm{jmr} .2006 .0088$.

(37) Calahorra, Y.; Kim, W.; Vukajlovic-Plestina, J.; Fontcuberta i Morral, A.; Kar-Narayan, S. Time-resolved open-circuit conductive atomic force microscopy for direct electromechanical characterisation. Nanotechnology 2020, 31 (40), 404003, DOI: 10.1088/1361-6528/ab9b4b.

(38) Zhao, M. H.; Wang, Z. L.; Mao, S. X. Piezoelectric characterization of individual zinc oxide nanobelt probed by piezoresponse force microscope. Nano Letters 2004, 4 (4), 587-590, DOI: 10.1021/n1035198a.

(39) Islam, R. A.; Priya, S. Realization of high-energy density polycrystalline piezoelectric ceramics. Applied Physics Letters 2006, 88 (3), DOI: 10.1063/1.2166201.

(40) Roscow, J. I.; Pearce, H.; Khanbareh, H.; Kar-Narayan, S.; Bowen, C. R. Modified energy harvesting figures of merit for stress- and strain-driven piezoelectric systems. European Physical Journal-Special Topics 2019, 228 (7), 1537-1554, DOI: 10.1140/epjst/e2019-800143-7.

(41) Whiter, R. A.; Narayan, V.; Kar-Narayan, S. A Scalable Nanogenerator Based on Self-Poled Piezoelectric Polymer Nanowires with High Energy Conversion Efficiency. Advanced Energy Materials 2014, 4 (18), 1400519, DOI: 10.1002/aenm.201400519.

(42) Crossley, S.; Kar-Narayan, S. Energy harvesting performance of piezoelectric ceramic and polymer nanowires. Nanotechnology 2015, 26 (34), DOI: 10.1088/0957-4484/26/34/344001.

(43) Calarco, R.; Stoica, T.; Brandt, O.; Geelhaar, L. Surface-induced effects in GaN nanowires. Journal of Materials Research 2011, 26 (17), 2157-2168, DOI: 10.1557/jmr.2011.211.

(44) Parida, S.; Sahoo, A. R.; Madapu, K. K.; Jaya, S. M.; Dhara, S. Role of the surface density of states in understanding size-dependent surface band bending in GaN nanowires. Applied Surface Science 2020, 510, DOI: 10.1016/j.apsusc.2020.145502.

(45) Calahorra, Y.; Yalon, E.; Ritter, D. On the diameter dependence of metal-nanowire Schottky barrier height. Journal of Applied Physics 2015, 117 (3), DOI: 10.1063/1.4906210.

(46) Lefki, K.; Dormans, G. J. M. MEASUREMENT OF PIEZOELECTRIC COEFFICIENTS OF FERROELECTRIC THIN-FILMS. Journal of Applied Physics 1994, 76 (3), 1764-1767, DOI: $10.1063 / 1.357693$.

(47) Zhang, Y.; Roscow, J.; Lewis, R.; Khanbareh, H.; Topolov, V. Y.; Xie, M. Y.; Bowen, C. R. Understanding the effect of porosity on the polarisation-field response of ferroelectric materials. Acta Materialia 2018, 154, 100-112, DOI: 10.1016/j.actamat.2018.05.007.

(48) Guo, R.; Wang, C. A.; Yang, A. K.; Fu, J. T. Enhanced piezoelectric property of porous lead zirconate titanate ceramics with one dimensional ordered pore structure. Journal of Applied Physics 2010, 108 (12), DOI: 10.1063/1.3525056. 
(49) Xu, T. T.; Wang, C. A. Grain Orientation and Domain Configuration in 3-1 Type Porous PZT Ceramics with Ultrahigh Piezoelectric Properties. Journal of the American Ceramic Society 2015, 98 (9), 2700-2702, DOI: 10.1111/jace.13725.

(50) Cha, S.; Kim, S. M.; Kim, H.; Ku, J.; Sohn, J. I.; Park, Y. J.; Song, B. G.; Jung, M. H.; Lee, E. K.; Choi, B. L.; Park, J. J.; Wang, Z. L.; Kim, J. M.; Kim, K. Porous PVDF As Effective Sonic Wave Driven Nanogenerators. Nano Letters 2011, 11 (12), 5142-5147, DOI: 10.1021/n1202208n. (51) Chandratre, S.; Sharma, P. Coaxing graphene to be piezoelectric. Applied Physics Letters 2012, 100 (2), DOI: $10.1063 / 1.3676084$.

(52) Zelisko, M.; Hanlumyuang, Y.; Yang, S. B.; Liu, Y. M.; Lei, C. H.; Li, J. Y.; Ajayan, P. M.; Sharma, P. Anomalous piezoelectricity in two-dimensional graphene nitride nanosheets. Nature Communications 2014, 5, DOI: 10.1038/ncomms5284.

(53) Datta, R.; Kappers, M. J.; Vickers, M. E.; Barnard, J. S.; Humphreys, C. J. Growth and characterisation of $\mathrm{GaN}$ with reduced dislocation density. Superlattices and Microstructures 2004, 36 (4-6), 393-401, DOI: 10.1016/j.spmi.2004.09.003. 